\title{
APPLICATION OF INDUCTION MODULE FOR ENERGY PERTURBATIONS IN THE UNIVERSITY OF MARYLAND ELECTRON RING*
}

\author{
B. Beaudoin", S. Bernal, I. Haber, R. Kishek, M. Reiser, K. Tian, J.C.T. Thangaraj, M. Walter, C. \\ Wu and P.G. O'Shea, IREAP, College Park, MD 20742, U.S.A.
}

\section{Abstract}

The University of Maryland Electron Ring (UMER) is a scaled storage ring using low-energy electrons to inexpensively model beams with high space-charge. With the ability to inject such beams comes the problem of longitudinal end erosion of both the head and tail. It is important therefore to apply suitably designed longitudinal focusing forces to confine the beam and prevent it from its normal expansion. This paper presents the design and prototyping of an induction cell for this purpose. Successful operation of the induction cell would push the achievable number of turns and also enable us to perform studies of the longitudinal physics of such highly space-charge dominated beams. The pulsed voltage requirements for such a system on UMER would require ear-fields that switch $3 \mathrm{kV}$ in about $8 \mathrm{~ns}$ or so for the most intense flat-top rectangular beam injected into the ring. This places a considerable challenge on the electronics used to deliver ideal waveforms with a compact module. Alternate waveforms are also being explored for other various injected beam shapes into UMER.

\section{INTRODUCTION}

The University of Maryland Electron Ring [1] is a lowenergy storage ring used to study highly space-charge dominated beams. UMER is a test bed for space charge dynamics of intense beams, such as those in X-ray FEL injectors [2], pulsed neutron sources [3], or heavy ion inertial fusion [4]. The machine has many capabilities for detailed experiments in both transverse and longitudinal dynamics such as, the capability of generating density perturbations either electrically [5], or by photo-emission [6]. A number of time-resolved diagnostics have been developed, such as Optical Transition Radiation (OTR) [7], fast phosphor screens [8], fast beam position monitors (BPMs) [9], and a fast high-resolution energy analyzer [10].

Longitudinal end erosion of both the head and tail is important for space charge dominated beams. After a number of turns, depending on the beam current, the beam will fill the ring with charge [11] and therefore properly applied axial focusing forces to confine the beam and prevent it from its expansion must be designed.

In this paper we will briefly describe some of the design work and considerations of an induction cell, and then show some preliminary results using the prototype induction module to apply velocity perturbations to the

\footnotetext{
*Work supported by U.S. Department of Energy grant numbers DE-FG02-94ER40855 and DE-F-G02-92ER54178, Office of Naval Research and the Joint Technology Office.

"beaudoin@umd.edu
}

UMER beam and finally compare the experimental results to the one dimensional cold fluid model.

\section{INDUCTION MODULE}

The basic principle of the Induction Module (IM) for beam acceleration, (see Fig. 1), is the use of a ferromagnetic isolator to minimize the short-circuit current that would otherwise flow into the laboratory structure while maximizing current delivered to the load [12], i.e. the beam. A lumped circuit diagram [13] is shown in (Fig. 2).

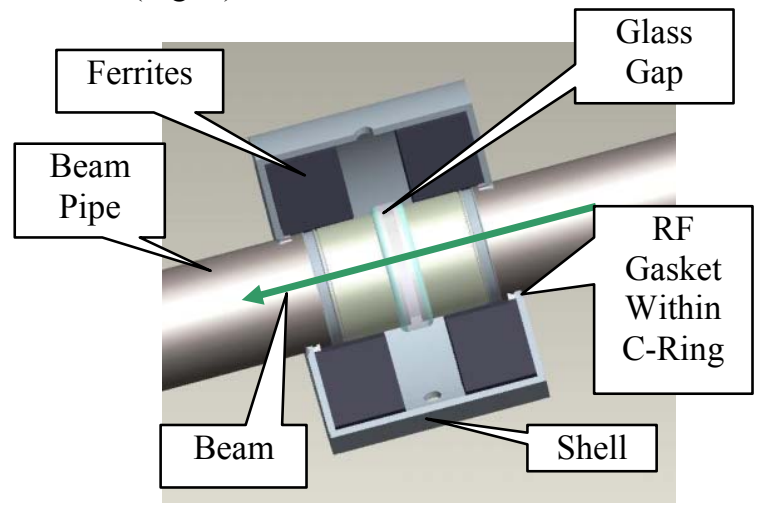

Figure 1: Pro-E drawing of the IM.

The ferrite cores are modeled by the inductor displayed in the basic circuit. The resistor terminates the transmission line from the pulser and finally the capacitor is a model of any stray capacitance that may reside in the cavity structure itself. The circuit has two current sources, the first being the pulsing electronics and the second is the beam current itself.

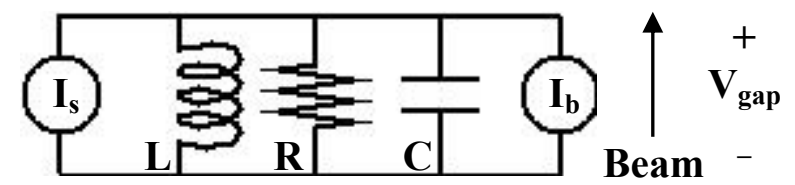

Figure 2: Circuit diagram of the IM.

The inductance of the ferrite cores installed in the prototype IM is approximately $24 \mathrm{uH}$, the terminating resistor is $50 \Omega$, and the stray capacitance was measured to approx $22 \mathrm{pF}$. One of the limiting factors of an induction cell, or any transformer for that matter, is the saturation of the ferrite material used for isolation or coupling [12]. Saturation in the IM will lead to a minimization of the load current and a maximization of the short circuit current [12]. We can calculate the full saturation limit of the cores by applying Faraday's law to the current loop around a single core in the cavity: 


$$
V_{\text {pulse }} \Delta t_{\text {pulse }}=\Delta B A_{\text {core }}
$$

where $\Delta \mathrm{t}_{\text {pulse }}$ is the pulse-width, $\mathrm{V}_{\text {pulse }}$ is the pulse voltage, $\Delta \mathrm{B}$ is the changing magnetic field in the core and $\mathrm{A}_{\text {core }}$ is the cross-sectional of the core.

The volt-second product in the equation above governs the flux swing from negative-to-positive saturation for an allowable pulse length $t_{\text {pulse }}$ and voltage $V_{\text {pulse }}$ applied to a given cross-section of material [14]. The difficulty that comes with pulsing the ferromagnetic material is the fact that after the cores are pulsed they must be reset by either using a DC negative bias to keep the ferrite in a reverse magnetization on the hysteresis loop or they can be pulsed with the opposite polarity to return the material to the center of the hysteresis loop [15]. Of course the duty cycle of the reset pulse and how large the $\mathrm{dB} / \mathrm{dt}$ is, must be considered when dealing with the power losses within the cores [15], such that you do not approach the curie temperature of the ferrous material.

In order to accomplish this, a bipolar pulsing circuit composed of two BEHLKE HTS 80-12-UF switches each connected in a capacitive discharge arrangement via a push-pull fashion was constructed. These switches, with a rise time of $2 \mathrm{nsec}$, are capable of withstanding voltages on the order of 8000 volts and peaks currents of up to 120 amps. The normalized circuit voltage for a negative pulse output across the $50 \mathrm{ohm}$ resistor in the circuit is displayed in (Fig. 3). Measurement was taken with an injected beam load of $20.78 \mathrm{~mA}$.

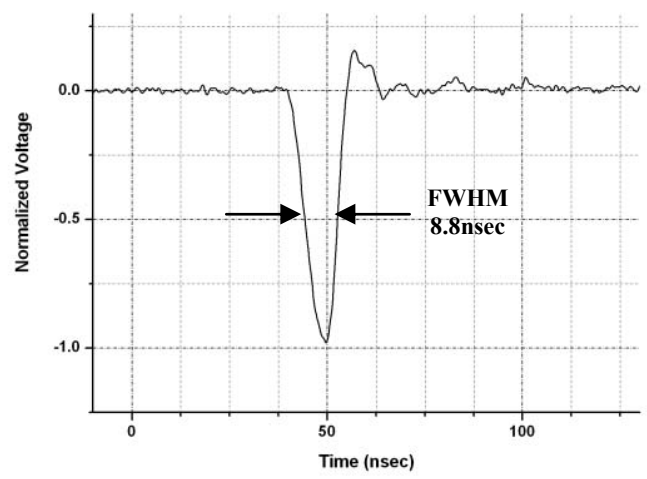

Figure 3: Negative pulse output voltage.

Loading of the gap voltage occurs when the induction module can not supply enough current to compete with the beam current. If we calculate the impedance of the circuit in (Fig. 2), in the limit where $\omega$ is large, such that the impedance of the inductor is large enough in comparison to the resistor that it dominates and the capacitance term is small enough to be negligible. We are left with the pulsed current source connected via a transmission line to a resistor $\mathrm{R}$. The gap voltage can be calculated from (Eq. 2).

$$
V_{\text {gap }}=\left(V_{\text {pulse }}-I_{b} Z_{o}\right) \frac{R}{R+Z_{o}}
$$

where $Z_{0}$ is the impedance of the transmission line between the module and electronics and $\mathrm{V}_{\text {gap }}$ is the voltage that appears across the resistor [16]. UMER has a maximum injected beam current of $100 \mathrm{~mA}$, so gap loading due to the beam current will reduce the applied pulse voltage at most by 5 volts, assuming $50 \mathrm{ohm}$ impedance.

\section{SPACE-CHARGE WAVES}

Beams are not perfect but often have fluctuations in energy or density. At the low-energy end of the machine, space charge can transform those fluctuations into space charge waves that propagate longitudinally along the beam. It is of interest to be able to deliberately generate perturbations and study their evolution. Perturbations may be applied to the UMER beam in a number of ways, either through photo induced current density perturbations [6] or electrically induced grid to cathode current density perturbations [5]. Energy perturbations may also be applied to the UMER beam through the use of the induction module.

By solving the one-dimensional fluid equations for a laminar beam [17] one will obtain the dispersion relation [17]. For small perturbations, such that the regime is still linear, the perturbation will form into two waves. A slow and fast wave traveling at a velocity called the "sound speed" $c_{s}[17]$ relative to the main beam velocity.

The perturbed beam velocity and current given an initial velocity or current perturbation are given by (Eq. 3 and 4),

$$
\begin{aligned}
& i_{1}(z, t)=-\frac{i_{o}}{2}\left[\delta \frac{v_{o}}{c_{s}}-\eta+(\eta-\delta) \frac{c_{s}}{v_{o}}\right] h\left(t-\frac{z}{v_{o}-c_{s}}\right) \\
& +\frac{i_{o}}{2}\left[\delta \frac{v_{o}}{c_{s}}+\eta+(\eta-\delta) \frac{c_{s}}{v_{o}}\right] h\left(t-\frac{z}{v_{o}+c_{s}}\right) \\
& v_{1}(z, t)=+\frac{v_{o}}{2}\left[\delta-(\eta-\delta) \frac{c_{s}}{v_{o}}\right] h\left(t-\frac{z}{v_{o}-c_{s}}\right) \\
& +\frac{v_{o}}{2}\left[\delta+(\eta-\delta) \frac{c_{s}}{v_{o}}\right] h\left(t-\frac{z}{v_{o}+c_{s}}\right)
\end{aligned}
$$

where $\delta$ is the initial strength of the velocity perturbation, $\eta$ is the initial strength of the current perturbation, $h(t)$ is a smooth unity function of the perturbation, $\mathrm{v}_{\mathrm{o}}$ and $\mathrm{i}_{\mathrm{o}}$ is the main beam velocity and current respectively [18].

\section{PERTURBATIONS}

The IM may be used to confine the beam longitudinally by correcting end erosion of the tail and or head of the beam via pulses designed to accelerate the tail and decelerate the head of the beam. The IM may also be used to place velocity perturbations or velocity tilts on the beam. We will briefly explain the experimental setup and procedure of placing velocity perturbations on the beam and then show some results and also compare the results 
to a one-dimensional simulation of the experiment in MATLAB.

The UMER beam propagates at a speed of $0.2 \mathrm{c}$, where $\mathrm{c}$ is the speed of light. There are three glass gaps in the ring equally spaced 3.83 meters apart where the first glass gap is 3.902 meters away from the cathode. The prototype induction module was installed on the first glass gap.

The 100nsec long beam is initially injected into the ring without any deliberate perturbations. As the middle of the beam approaches the induction module, the cell is pulsed with either a positive or negative pulse. After which, the four plates of the following BPMs, from RC 5 through RC 13 , are summed together for a total current measurement around the ring as the beam and perturbation propagate. Experimental data for a peak $-300 \mathrm{eV}$ perturbation is displayed in the figure below, along with the 1-D simulation of the perturbation.

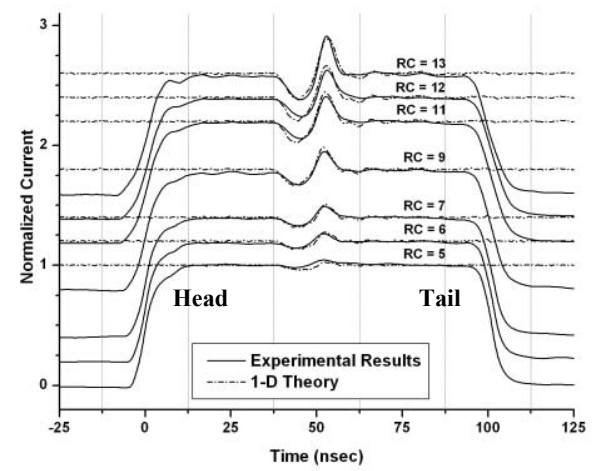

Figure 4: Comparison of experimental and 1-D simulation data for a $-300 \mathrm{eV}$ pulse perturbation.

A negative pulse perturbation is represented by an axial E-field vector in the direction of beam propagation. The applied energy modulation pushes electrons backward from the perturbed point, forcing a density increase in the opposite direction of beam propagation and a density dip in the other direction. As the beam propagates, the perturbation will split into a slow and fast wave [18].

The applied voltage pulse captured from the oscilloscope was used in the 1-D cold fluid model (see Fig. 3), for a peak $-300 \mathrm{eV}$ field and calculated for the same ring chamber distances as in the experimental data then plotted in figure 4 as the dotted curves.

The model assumes small perturbations, so naturally there is good agreement with the 1-D theory at low peak fields for both negative and positive perturbations. As the peak field was increased to $600 \mathrm{eV}$, non-linear effects become apparent in comparison to a linear model. The experimental and simulation data for a peak $-600 \mathrm{eV}$ perturbation is shown in (Fig. 5).

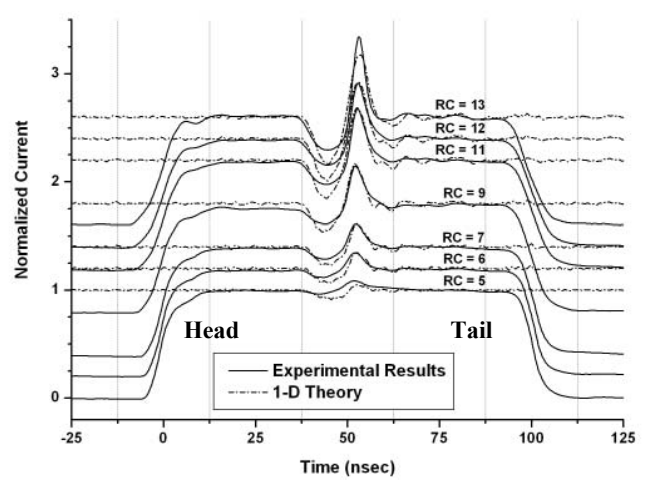

Figure 5: Comparison of experimental and 1-D simulation data for a $-600 \mathrm{eV}$ pulse perturbation.

\section{SUMMARY}

The induction cell brings in a new set of experiments into UMER's vast repertoire which will allow us to explore other concepts in the current framework.

\section{ACKNOWLEDGEMENTS}

We wish to thank all who assisted this work supported by DOE, ONR and the Joint Technology Office.

\section{REFERENCES}

[1] R. Kishek, "These Proceedings," PAC07, 2007.

[2] P. McIntosh, "Overview of the RF systems for LCLS," PAC05, 2005.

[3] R. Kustom, "Intense Pulsed Neutron Sources," IEEE Nuclear Science, 3, 28, (1981).

[4] J. Kwan, "High Current Density Ion Sources for Heavy Ion Fusion Accelerators,” IEEE, p.2755, 1998.

[5] K. Tian, PRST-AB, 014201 (2006).

[6] J.C.T. Thangaraj, "These Proceedings," PAC07, 2007.

[7] R. Fiorito, "These Proceedings," PAC07, 2007.

[8] K. Tian, "These Proceedings," PAC07, 2007.

[9] B. Quinn et al., "Design and Testing of a Fast Beam Position Monitor," Proc. 2003 PAC.

[10] Y. Cui et al., Rev. Sci. Instrum. 75, 2736 (2004).

[11] A. Faltens, J. Appl. Phys. 61 (12), 15 June 1987.

[12] S. Humphries, Principles of Charged Particle Acceleration, (Wiley, New York, 1986).

[13] J.J. Deng et al., "Longitudinal Focusing of SpaceCharge Dominated Beams in the UMD Electron Ring," PAC97, 1997.

[14] C. Sullender, IEEE Power Electronics, 8, 1, (1993).

[15] W. Waldron, Private Communication, January 2007.

[16] G. Caporaso, Private Communication, January 2007.

[17]M. Reiser, Theory and Design of Charged Particle Beams (Wiley, New York, 1994).

[18] J.G. Wang, et al., Phys. Rev. Lett., 71, 1836, (1993). 